\title{
THE OCCURRENCE OF LUMBOSACRAL TRANSITIONAL VERTEBRA IN TWO HUNTING BREEDS OF DOGS ITS ASSOCIATION WITH HIP DYSPLASIA
}

\author{
M. KURICOVÁ, T. LIPTÁK and V. LEDECKÝ \\ University Teaching Hospital, Medipark, Small Animal Clinic, Department of Surgery, \\ Orthopaedics, Radiology and Reproduction, University of Veterinary Medicine and \\ Pharmacy, Komenskeho 73, 04001 Kosice, Slovakia
}

Received: 30 September 2018; Accepted: 30 October 2018

\begin{abstract}
The lumbosacral transitional vertebra (LTV) has become the topic of discussions of veterinarians, breeders and owners of the dogs, especially in connection with genetics and its possible impact on canine hip joint dysplasia (HD). The aim of this study was to evaluate the occurrence of LTV in dogs examined for HD at one clinic during the years 2016 and 2017. We assessed the possible connection between these two diseases in two hunting breeds: Bavarian Mountain Dog and Hanoverian Mountain Dog. There is only little evidence, when compared to some other large breed dogs, on occurrence of the LTV in mentioned hunting breeds. These two breeds are the most common among hunting dogs undergoing HD screening in Slovakia, in addition, they are unlikely affected with clinical signs of the cauda equina entrapment. The Cauda equina syndrome is the common clinical manifestation of lumbosacral transitional vertebra and this can have negative impact on the mobility and quality of life. In this study, the incidence of lumbosacral transitional vertebra in the investigated group of 169 dogs was $66.86 \%$. The LTV type 1 was present in $36.09 \%$ of dogs, LTV type 2 was present in $18.34 \%$ of dogs and LTV type 3 in $12.43 \%$ of dogs. The direct relationship between occurrence of LTV and HD was not confirmed.
\end{abstract}

Key words: Dog, lumbosacral transitional vertebra, hip dysplasia, genetic bone diseases.

\section{INTRODUCTION}

The lumbosacral transitional vertebra (LTV) is a common congenital anomaly of the spine of the dogs. It is an abnormally shaped vertebra, occurring most often in the thoracolumbar or lumbosacral segment of the spine. Changes of the last lumbar vertebra or the first sacral vertebra can cause its asymmetric connection with the pelvis and it may be related to the changes of number of vertebrae or rather their morphology. This transitional vertebra reduces the stability of a given spinal segment and predisposingly influences the development of degenerative diseases of the lumbosacral joint and the intervertebral disc between the changed vertebrae (Colter 1993, Wheeler 1994, Damur-Djuric et al., 2006, Westwort and Sturges 2010). Since an asymmetrically formed LTV may affect the uneven bilateral hip joint load, its occurrence may be related with the occurrence of hip dysplasia. An LTV can lead to rotation of the pelvis, which favours an

Corresponding author: M. KURICOVÁ

E-mail address: maria.kuricova@uvlf.sk

Present address: University Teaching Hospital, Medipark, Small Animal Clinic, Department of Surgery, Orthopaedics, Radiology and Reproduction, University of Veterinary Medicine and Pharmacy, Komenskeho 73, 04001 Kosice, Slovakia abnormal development of the hip joint (Morgan et al., 2000, Flückiger et al., 2017). Italso influences the mobility and physical activity and the emergence of Cauda equina syndrome. This anomaly affects especially large breeds of dogs (Damur-Djuric et al., 2006). From the studies published so far, it can be concluded that the different occurrence of LTV in different breeds of dogs can have a genetic basis and hence a hereditary character (Morgan et al., 1999, Damur-Djuric et al., 2006, Wigger et al., 2009).

The Orthopedic Foundation for Animals (OFA), as the world's most recognized breeders' organization, has also begun to monitor the occurrence of a lumbosacral transitional vertebra in dogs. So far, it has issued the recommended measures for breeders to refer to the breeding of two individuals in the case of a transitional lumbosacral vertebra (http://www. ofa.org/hd_procedures.html). The occurrence of lumbosacral transitional vertebra in dogs was first described by J. P. Morgan in 1968. The incidence of this disease in the dog population is $3.5 \%$ according to Damur-Djuric et al. (2006). In Germany, during the HD screening of the German shepherd dogs, an LTV was detected in one older study in $8-11 \%$ of the cases (Morgan et al., 1993). In a study in the Great Pyrenees dog, the incidence was $1.5 \%$ and in the Labrador Retriever $1.8 \%$ of the investigated dogs 
(Morgan et al., 1985). One study even reports the $29 \%$ prevalence in German shepherd dogs. In this study, the isolation of spinous process of S1 and S2 was considered LTV and the prevalence was $78 \%$ of cases (Wigger 2009).

According to Bailey and Morgan (1992), the presence of a lumbosacral transitional vertebra is painless and this develops mostly asymptomatically. However, later studies indicate its effect on the biomechanics of the lumbosacral and sacroiliacal junction (Hanna 2001), which may subsequently cause degeneration of the intervertebral disc or lead to the Cauda equina syndrome. These clinical symptoms develop for a long time and are of a chronic nature prior to manifestation. Weakness of the pelvic limbs, pain and lameness will be reflected with the development of degenerative changes (Morgan et al., 1999, Morgan et al., 2000, Damur-Djuric et al., 2006).

Diagnosis of the LTV is performed by radiographic examination on the ventrodorsal (asymmetric LTV with the pelvis) and possibly laterolateral projection (if a change in the number of vertebrae is assumed). Due to the many variations in the morphology of the transitional lumbosacral vertebra, different systems of its evaluation have evolved over time. In the veterinary medicine, four are applied, which are named after their authors (Breit et al., Künzel 2003, Damur-Djuric 2006, Flückiger et al., 2009, Lappalainen 2012).

We have focused on the comparison of individual types of transitional lumbosacral vertebrae in individual groups of dogs divided by degree of hip dysplasia, and vice versa. The hypothesis was that in dogs with hip dysplasia, the LTV will occur more frequently than in dogs without HD, and dogs with a higher degree of dysplasia may have more asymmetrical types of LTV.

\section{MATERIALS AND METHODS}

This study included 169 dogs, which have undergone radiographic hip dysplasia (HD) examination according to the Federation Cynologique Internationale (FCI) standard protocol for official examination of canine HD in potentially breeding individuals. The radiographs were obtained from the archive of the Small Animal Clinic during years 2016 and 2017 from all assessed dogs, the assessment of HD was performed by skilled official evaluator on standard radiographs in ventrodorsal position. After obtaining complete data we evaluated the occurrence of different types of transitional lumbosacral vertebra in these dogs according to Flückiger et al. (2009).

We used the Spearman correlation to evaluate the correlation between the LTV and HD.

\section{RESULTS}

In the group of 169 dogs, there were 155 Bavarian mountain dogs and 14 Hanoverian mountain dogs in the age between one year and eight years, with the mean age of $1.68 \pm 1.05$ (mean \pm SD). After collecting the radiographs, we determined the occurrence and the type of the transitional lumbosacral vertebra in individual dogs. Out of the 169 evaluated dogs, $64(37.87 \%)$ dogs had different degrees of hip dysplasia. The lumbosacral transitional vertebra was diagnosed in 113 dogs $(66.86 \%)$.

The investigated group of dogs was then subdivided into four groups according to the presence and type of the transitional lumbosacral vertebra. Subgroups based on the LTV type according to Flückiger et al. (2009) as follows:

$\rightarrow$ Group LTV 0 included 56 dogs (33.14\%) without transitional lumbosacral vertebra

$\rightarrow$ Group LTV 1 consisted of 61 dogs (36.09\%) with unrelated processus spinosus $\mathrm{S} 1$ and $\mathrm{S} 2$

$\rightarrow$ Group LTV 2 included 31 dogs (18.34\%) with a separation of the transitional lumbosacral vertebra from the os sacrum and a symmetric malformation of processus transversi

$\rightarrow$ Group LTV 3 consisted of 21 dogs (12.43\%) with asymmetrically formed processus transversus and unrelated processus spinosus of the LTV from the processus spinosus of the sacral bone.

The incidence of LTV in the investigated group of 169 dogs was $66.86 \%$. Of the whole evaluated group, LTV type 1 was present in $36.09 \%$ of dogs, LTV type 2 was found in $18.34 \%$ of dogs and LTV type 3 in $12.43 \%$ of dogs. Other $33,14 \%$ of dogs were without the presence of LTV. Of the 169 dogs examined, 105 $(62.13 \%)$ had no hip dysplasia and 41 (39.05\%) of them did not have LTV. Type 1 LTV was present in $33(31.43 \%)$ dogs from the subgroup without HD. Type 2 LTV was present in 19 (18.09\%) dogs without HD and LTV type 3 was present in 12 (11.43\%) dogs without dysplasia. The group with borderline HD consists of 42 dogs, of which 13 (30.95\%) did not present LTV. Type 1 LTV was present in 19 dogs (45.24\%), LTV type 2 was found in $7(16.67 \%)$ dogs and LTV type 3 in only $3(7.14 \%)$ dogs with borderline HD. A subgroup of dogs with mild dysplasia consists of 21 dogs, of which only 2 were (9.52\%) without LTV. However, type 1 LTV was present in $8(38.10 \%)$ dogs, LTV type 2 in 5 (23.81\%) dogs, and LTV type 3 in $6(28.57 \%)$ dogs. There was only one dog with moderate HD in our study, and this was the case with the presence of the type $1 \mathrm{LTV}$. 
This study has not demonstrated a clear correlation between the presence of transitional lumbosacral vertebra and the development of hip dysplasia $(r=$ 0.24 ), but we have seen a high prevalence of LTV type 1 in the evaluated group. Out of 113 dogs in which a LTV was present, this type of LTV with unconnected processus spinosus of the first two sacral vertebrae was found in $61 \mathrm{dogs}(53.98 \%)$ of cases.

\section{DISCUSSION}

Based on the research done so far, it can be concluded that not all dogs positive for hip dysplasia also have a transitional lumbosacral vertebra, and not all LTV positive dogs are positive for HD. However, most of these studies were based on radiographic diagnostics only in the ventrodorsal position in focusing on the HD assessment and thus did not have to reveal the LTV present in symmetric form. We can only diagnose this in a laterolateral position taking into account the LTV in the form of the eight lumbar vertebra. Only if processus spinosus of S1 is clearly separated from the following two sacral vertebrae, or if it is not in a linear position, then we can also diagnose this anomaly on the VD projection. The asymmetric development of LTV acts on the asymmetric development of the pelvis in the horizontal plane, which can directly affect the forming of the shape and depth of the acetabulum. On the radiograph we observe the wider wings of iliac bone on the side of the stronger connection of the LTV with the corresponding part of the iliac bone. This suggests that the connection between sacral bone and the pelvis can directly affect the joint cavity formation and consequently the development of canine hip dysplasia (Morgan et al., 2000).

The aim of this study was to investigate the effect of the presence of LTV on the occurrence of hip dysplasia in dogs. The hypothesis was that a higher degree of transitional vertebra may result in the development of a higher degree of hip dysplasia. Because dogs are being investigated at a young age, it is possible that the HD might be confirmed at a later age and predisposed individuals should be radiographed after some times during the working life.

Abnormal hip joint development is a common degenerative hip joint disease in dogs (Smith et al., 2012), but also in cats (Holt 1978, Patsikas et al., 1998), and its aetiology includes multiple genes and environmental factors. Environmental factors alone do not cause dysplasia of the hips, but the affected animals are genetically predisposed (Necas 2004). From the publications that have been published so far, $5 \%$ to $35 \%$ of examined dogs are HD affected, with a heritability rate of 0.2-0.6. At birth, hip joints usually appear normal and later become dysplastic. The first change is the hip joint laxity when the femoral head is not well established in the acetabulum. This leads to abnormal joint load, resulting in bone remodelling and degenerative joint disease. This can affect any breed of dogs or cats. The highest occurrence is in dogs of large and giant breeds and purebred cats (Muhlbauer, Kneller 2013). A high representation of dogs without hip dysplasia could be largely caused by long-term breeding of these hunting breeds since the inclusion of potential breeding individuals in breeding is based on compliance with the FCI criteria, including the results of imaging diagnostics. Diagnosis of HD is made from the ventrodorsal radiographs with a maximum extension of the pelvic legs in the caudal direction, with an emphasis on reaching the symmetrical position of the pelvis, so that the whole pelvis is caught on the X-ray image, with parallel femoral bones and patella located in the middle of the epicondyles of the distal part of the femur. In some cases of LTV, there is often not possible to get really symmetric VD position because of the anatomical abnormalities and rotation of the pelvis. The results of our study are comparable to those found by Wigger et al. (2012). It remains nonproven that separation of the spinal dorsal process of $\mathrm{S} 1$ is a mild form of a true LTV. Recent data suggest that this feature is overread on plain radiographs (Lappalainen et al., 2012), which may also a limitation of the study. Angulation and deformation of the LVT in one study was seen more frequently in dogs with an asymmetrical LTV. Preferentially, the LTV was angulated towards the side of the worse hip joint. An asymmetrical LTV does not always elicit rotation of the pelvis, indicating that the causative factors of malalignment between the LTV and pelvis are complex and have not been fully revealed yet (Flückiger et al., 2017). Radiographs made in lateral positioning can help to discriminate the cause of rotation, but this is not a part of the official FCI hip examination. Computed tomography imaging may help to identify the true cause of malalignment, but computed tomography is not an option for mass screening (Lappalainen et al., 2012).

\section{ACKNOWLEDGEMENT}

This work was supported by the financial support from the Scientific Grant Agency of Ministry of Education, Science, Research and Sport of the Slovak Republic and the Slovak Academy of Sciences (VEGA No. 1/0898/15).

\section{REFERENCES}

Bailey, C.S. and Morgan, J.P. (1992): Congenital spinal malformations. Vet. Clin. North Amer. Small Anim. Pract., 22, 4: 985-1015.

Breit, S.; Knaus, I. and Künzel, W. (2003): Differentiation between lumbosacral transitional vertebrae, pseudolumbarisation, and lumbosacral osteophyte formation in 
ventrodorsal radiographs of the canine pelvis. Vet. J., 165, 1: 36-42.

Colter, S.B. (1993): Congenital anomalies of the spine. Disease mechanisms in small animal surgery, 950-959.

Damur-Djuric, N.; Steffen, F. and Hässig, M. (2006): Lumbosacral transitional vertebrae in dogs: classification and prevalence in various breeds. Vet Radiol Ultrasound, 47: 32-38.

Flückiger, M.A.; Steffen, F.; Hässig, M. and Morgan, J.P. (2017): Asymmetrical lumbosacral transitional vertebrae in dogs may promote asymmetrical hip joint development. Vet. Comp. Orthop. Traum., 30, 2: 137-142.

Flückiger, M.; Geissbühler, U. and Lang, J. (2009): Lumbosakrale Übergangswirbel: Welche Bedeutung haben sie für die Gesundheit von betroffenen Hunden?. Schweizer Archiv für Tierheilkunde, 151, 3: 133-135.

Hanna, F.Y. (2001): Lumbosacral osteochondrosis: radiological features and surgical management in 34 dogs. J. Small Anim. Pract., 42, 6: 272278.

Holt, P.E. (1978): Hip dysplasia in a cat. J. Small Anim. Pract., 12: 273-276.

Komsta, R.; Łojszczyk-Szczepaniak, A. and Dębiak, P. (2015): Lumbosacral transitional vertebrae, canine hip dysplasia, and sacroiliac joint degenerative changes on ventrodorsal radiographs of the pelvis in police working German Shepherd dogs. Topics in companion animal medicine, 30, 1: 10-15.

Lappalainen, A.K.; Salomaa, $R$. and Junnila J. (2012): Alternative classification and screening protocol for transitional lumbosacral vertebra in German Shepherd dogs. Acta Vet. Scand., 54: 27-37.

Morgan, J.P.; Wind, A. and Davidson, A.P. (2000): Hereditary Bone and Joint Diseases in the Dog: Osteochondroses, Hip Dysplasia, Elbow
Dysplasia. Hannover: Schlütersche. p. 109208.

Morgan, J.P. (1999): Transitional lumbosacral vertebral anomaly in the dog: a radiographic study. J. Small Anim. Pract., 40: 167-172

Morgan, J.P. (1993): Lumbosacral transitional vertebrae as a predisposing cause of cauda equina syndrome in German shepherd dogs: 161 cases (1987-1990). J. Am. Vet. Med. Assoc., 202, 11: 1877-1882.

Morgan, J.P. (1985): Radiographic diagnosis and control of canine hip dysplasia. Iowa State University Press.

Muhlbauer, M.C. and Kneller, S.K. (2013): Radiography of the $\mathrm{dog}$ and cat: guide to making and interpreting radiographs. John Wiley \& Sons.

Nečas, A. (2004): Diagnosis and therapy of the hip dysplasia and elbow dysplasia. Ed. Veterinary and Pharmaceutical University, Brno, p. 64, ISBN: 8073054833.

Patsikas, M.N. (1998): Hip dysplasia in the cat: a report of three cases. J. Small Anim. Pract., 39, 6: 290-294.

Smith, G.K. (2012): Chronology of hip dysplasia development in a cohort of 48 Labrador retrievers followed for life. Vet. Surg., 41, 1: 20-33.

Westworth, D.R. and Sturges, B.K. (2010): Congenital spinal malformations in small animals. Vet. Clinics: Small Anim. Pract., 40, 5: 951-981.

Wheeler, S.J. (1994): Small animal spinal disorders, diagnosis and surgery. Mosby Wolfe Publishers.

Wigger, A.; Julier-Franz, Ch. and Tellhelm, B. (2009): Lumbosakraler Übergangswirbel beim Deutschen Schäferhund: Häufigkeit, Formen, Genetik und Korrelation zur Hüftgelenksdysplasie. Tieraerztl. Prax., 37(K): 7-13. 